Bull. Austral. Math. Soc.

47 A55, 47L20

VOL. 64 (2001) [233-243]

\title{
ON THE OPERATORS WHICH ARE INVERTIBLE MODULO AN OPERATOR IDEAL
}

\author{
Pietro Aiena, Manuel González And Antonio Martínez-Abejón
}

We study the semigroups $\mathcal{A}_{l}$ and $\mathcal{A}_{r}$ of left and right invertible operators modulo an operator ideal $\mathcal{A}$, respectively. We show that these semigroups allow us to obtain useful characterisations of the radical $\mathcal{A}^{\mathrm{rad}}$ of $\mathcal{A}$. For example, $\mathcal{A}^{\mathrm{rad}}$ is the perturbation class for $\mathcal{A}_{l}$ and $\mathcal{A}_{r}$.

\section{INTRODUCTION}

Atkinson [3] studied the operators which are left invertible $\Phi_{l}(X, Y)$ or right invertible $\Phi_{r}(X, Y)$ modulo $\mathcal{K}$, with $\mathcal{K}$ the compact operators. He proved that an operator $T \in \mathcal{L}(X, Y)$ belongs to $\Phi_{l}$ or $\Phi_{r}$ if and only if the kernel and the range of $T$ are complemented and additionally, the kernel is finite dimensional or the range is finite codimensional, respectively. Yood [19] obtained some perturbation results for these classes and Lebow and Schechter [12] proved that the inessential operators form the perturbation class for $\Phi_{l}(X)$ and $\Phi_{r}(X)$.

Yang [18] extended some results of $[3,19]$ to operators invertible modulo $\mathcal{W}$, with $\mathcal{W}$ the weakly compact operators. His aim was to study a generalised Fredholm theory in which the reflexive spaces played the role of the finite dimensional spaces. Moreover, Astala and Tylli [4] compared the left-invertible operators modulo $\mathcal{W}$ with the Tauberian operators and other classes of operators defined in terms of weak compactness.

In this paper we study the classes $\mathcal{A}_{l}$ and $\mathcal{A}_{\boldsymbol{r}}$ of operators which are left and right invertible respectively, modulo an operator ideal $\mathcal{A}$. We show that these classes are open semigroups in the sense of [2], and that there is a close connection between $\mathcal{A}_{l}, \mathcal{A}_{r}$ and the radical $\mathcal{A}^{\mathrm{rad}}$ (in the sense of [15]) of the operator ideal $\mathcal{A}$. In fact, if $\mathcal{A}, \mathcal{B}$ are operator ideals, then the equalities $\mathcal{A}^{\mathrm{rad}}=\mathcal{B}^{\mathrm{rad}}, \mathcal{A}_{l}=\mathcal{B}_{l}$ and $\mathcal{A}_{r}=\mathcal{B}_{r}$ are equivalent. We obtain characterisations of $\mathcal{A}^{\text {rad }}$ simpler than the original definition in [15] and we show that $\mathcal{A}^{\text {rad }}$ is the perturbation class for $\mathcal{A}_{l}, \mathcal{A}_{l}$ and $\mathcal{A}_{l} \cap \mathcal{A}_{r}$.

Received 21st November, 2000

The first author was supported by the International Cooperation Project in Mathematics between the CONICIT (Venezuela) and the CNR (Italy). The second and third authors were supported in part by the DGESIC (Spain), Grant PB 97-0349.

Copyright Clearance Centre, Inc. Serial-fee code: 0004-9727/01 \$A2.00+0.00. 
Denoting by $\mathcal{W}$ the weakly compact operators, we compare $\mathcal{W}_{l}$ with $\Phi_{l}$ and $\mathcal{W}_{r}$ with $\Phi_{r}$. They coincide in $\mathcal{L}(X, Y)$ when one of the spaces $X, Y$ has the Dunford-Pettis property. Moreover, we give several examples of operator ideals $\mathcal{A}$ which are maximal in the sense that $\mathcal{B} \subset \mathcal{A}$ for each operator ideal $\mathcal{B}$ such that $\operatorname{Sp}(\mathcal{A})=\operatorname{Sp}(\mathcal{B})$; in particular, $\mathcal{A}=\mathcal{A}^{\mathrm{rad}}$. Observe that it is not known whether $\mathcal{K}^{\mathrm{rad}}$ is maximal in this sense $([\mathbf{1 5}$, 4.3.7]).

In this paper paper, $X, Y$ and $Z$ are Banach spaces and $X^{*}$ is the dual space of $X$. Also $\mathcal{L}(X, Y)$ is the set of all (continuous linear) operators from $X$ into $Y$. For $T \in \mathcal{L}(X, Y), N(T)$ and $R(T)$ are the kernel and the range of $T$, and $T^{*} \in \mathcal{L}\left(Y^{*}, X^{*}\right)$ is the conjugate operator of $T$.

Given a closed subspace $M$ of $X$, we denote by $J_{M}$ the inclusion of $M$ into $X$, and by $Q_{M}$ the quotient map from $X$ onto $X / M$. A subspace $M$ of $X$ is complemented if it is the range of a continuous linear projection on $X$. Of course, complemented subspaces are closed.

We denote by $\mathcal{L}$ the class of all operators between Banach spaces; that is, the union of all the sets $\mathcal{L}(X, Y)$, and by $\mathcal{F}$ and $\mathcal{G}$ the subclasses of all finite rank and all bijective operators, respectively. Given a subclass $\mathcal{A} \subset \mathcal{L}$, the components of $\mathcal{A}$ are the subsets

$$
\mathcal{A}(X, Y):=\mathcal{A} \cap \mathcal{L}(X, Y)
$$

We write $\mathcal{A}(X)$ in the case $X=Y$.

DEFinition 1.1: ([15]) A subclass $\mathcal{A} \subset \mathcal{L}$ is an operator ideal if it satisfies the following conditions:

$\left(\alpha_{1}\right) \mathcal{F} \subset \mathcal{A}$.

$\left(\alpha_{2}\right) \quad \mathcal{A}(X, Y)$ is a subspace of $\mathcal{L}(X, Y)$.

$\left(\alpha_{3}\right)$ If $B \in \mathcal{L}(W, X), T \in \mathcal{A}(X, Y)$ and $A \in \mathcal{L}(Y, Z)$, then $A T B \in \mathcal{A}(W, Z)$.

Every operator ideal $\mathcal{A}$ has associated a space ideal $\operatorname{Sp}(\mathcal{A})$, given by

$$
\operatorname{Sp}(\mathcal{A}):=\left\{X: \text { the identity } I_{X} \in \mathcal{A}\right\} \text {. }
$$

The dual operator ideal $\mathcal{A}^{d}$ of $\mathcal{A}$ is defined by $\mathcal{A}^{d}=\left\{T \in \mathcal{L}: T^{*} \in \mathcal{A}\right\}$. Clearly $\operatorname{Sp}\left(\mathcal{A}^{d}\right)=\left\{X: X^{*} \in \operatorname{Sp}(\mathcal{A})\right\}$.

EXAMPLES OF OPERATOR IDEALS. ([15]) Let $T \in \mathcal{L}(X, Y)$.

$\mathcal{K}$ compact operators: $T \in \mathcal{K}$ if it takes bounded sets into relatively compact sets.

$\mathcal{S S}$ strictly singular operators: $T \in \mathcal{S}$ if there is no infinite dimensional closed subspace $M$ of $X$ so that the restriction $T \mid M$ is an isomorphism.

$\mathcal{W}$ weakly compact operators: $T \in \mathcal{W}$ if it takes bounded sets into relatively weakly compact sets.

$\mathcal{C C}$ completely continuous operators: $T \in \mathcal{C C}$ if it takes weakly Cauchy sequences into convergent sequences. 
$\mathcal{U C}$ unconditionally converging operators: $T \in \mathcal{U C}$ if it takes weakly unconditionally Cauchy series into unconditionally convergent series.

The space ideals of $\mathcal{K}, \mathcal{W}, \mathcal{C C}$ and $\mathcal{U C}$ are the finite dimensional spaces, the reflexive spaces, the spaces with the Schur property and the spaces that contain no subspaces isomorphic to $c_{0}$, respectively.

The following concept of semigroup was introduced in [2].

DEFINITION 1.2: A subclass $\mathcal{S} \subset \mathcal{L}$ is an operator semigroup (a semigroup, for short) if it satisfies the following conditions:

$\left(\sigma_{1}\right) \mathcal{G} \subset \mathcal{S}$.

$\left(\sigma_{2}\right) \quad S \in \mathcal{S}(W, Y)$ and $T \in \mathcal{S}(X, Z)$ if and only if $S \oplus T \in \mathcal{S}(W \oplus X, Y \oplus Z)$.

$\left(\sigma_{3}\right) \quad$ If $T \in \mathcal{S}(X, Y)$ and $S \in \mathcal{S}(Y, Z)$, then $S T \in \mathcal{S}(X, Z)$.

EXAMPles of SEMIgRoups. [2] Every operator ideal $\mathcal{A}$ has associated two semigroups $\mathcal{A}_{+}$and $\mathcal{A}_{-}$, given by

$$
\mathcal{A}_{+}:=\{T \in \mathcal{L}: S \in \mathcal{L}, T S \in \mathcal{A} \Rightarrow S \in \mathcal{A}\}
$$

that is, $T \in \mathcal{A}_{+}(X, Y)$ if and only if for every $Z$ and every $S \in \mathcal{L}(Z, X)$, we have $T S \in \mathcal{A}$ implies $S \in \mathcal{A}$. Analogously,

$$
\mathcal{A}_{-}:=\{T \in \mathcal{L}: S \in \mathcal{L}, S T \in \mathcal{A} \Rightarrow S \in \mathcal{A}\} .
$$

The semigroups $\mathcal{K}_{+}, \mathcal{K}_{-}, \mathcal{W}_{+}$and $\mathcal{W}_{-}$coincide with the upper semi-Fredholm, lower semi-Fredholm, Tauberian $([\mathbf{1 0}])$ and cotauberian operators $([\mathbf{1 7}])$, respectively. The semigroups $\mathcal{U} \mathcal{C}_{+}$and $\mathcal{U C} \mathcal{C}_{-}$were studied in [8]. We refer to [2] for further results.

DEFINITION 1.3: Let $X$ and $Y$ be Banach spaces, and let $\mathcal{S}$ be a semigroup such that $\mathcal{S}(X, Y)$ is non-empty.

The component $P \mathcal{S}(X, Y)$ of the perturbation class $P \mathcal{S}$ of $\mathcal{S}$ is defined by

$$
P \mathcal{S}(X, Y):=\{K \in \mathcal{L}(X, Y): T+K \in \mathcal{S}(X, Y) \text { for every } T \in \mathcal{S}(X, Y)\} .
$$

PROPOSITION 1.4. ([12, 2]) Let $\mathcal{S}$ be a semigroup. Then

(a) $P \mathcal{S}(X, Y)$ is a subspace of $\mathcal{L}(X, Y)$ and $P \mathcal{S}(X)$ is a two-sided ideal in $\mathcal{L}(X)$.

(b) If $\mathcal{S}(X, Y)$ is an open subset of $\mathcal{L}(X, Y)$, then $P \mathcal{S}(X, Y)$ is closed.

An even more general concept of perturbation class was introduced by Lebow and Schechter [12].

\section{MAIN RESUltS}

In this section $\mathcal{A}$ is an operator ideal. We introduce and study the semigroups $\mathcal{A}_{l}$ and $\mathcal{A}_{\tau}$ of operators which are left or right invertible modulo $\mathcal{A}$. 
Definition 2.1: Let $T \in \mathcal{L}(X, Y)$. We define the classes $\mathcal{A}_{l}$ and $\mathcal{A}_{T}$ as follows:

$T \in \mathcal{A}_{l}$ if there exists $A \in \mathcal{L}(Y, X)$ such that $I_{X}-A T \in \mathcal{A}(X)$.

$T \in \mathcal{A}_{\boldsymbol{r}}$ if there exists $B \in \mathcal{L}(Y, X)$ such that $I_{Y}-T B \in \mathcal{A}(Y)$.

Let us denote by $\mathcal{R} \mathcal{R}$ the relatively regular operators $([\mathbf{3}])$; that is, the operators with complemented kernel and range. The relatively regular operators provide basic examples of operators in $\mathcal{A}_{l}$ and $\mathcal{A}_{r}$.

EXAMPLES 2.2 .

(a) Let $T \in \mathcal{L}(X, Y)$. Then

$$
\begin{gathered}
T \in \mathcal{K}_{l} \Longleftrightarrow T \in \mathcal{R} \mathcal{R} \text { and } \operatorname{dim} N(T)<\infty \\
T \in \mathcal{K}_{r} \Longleftrightarrow T \in \mathcal{R} \mathcal{R} \text { and } \operatorname{dim} Y / R(T)<\infty .
\end{gathered}
$$

(b) Let $T \in \mathcal{R} \mathcal{R}(X, Y)$. Then

$$
N(T) \in \operatorname{Sp}(\mathcal{A}) \Longleftrightarrow T \in \mathcal{A}_{l} \quad \text { and } \quad Y / R(T) \in \operatorname{Sp}(\mathcal{A}) \Longleftrightarrow T \in \mathcal{A}_{r},
$$

but Example (c) below shows that neither $\mathcal{A}_{l}$ nor $\mathcal{A}_{r}$ is contained in $\mathcal{R} \mathcal{R}$ in general.

(c) If $X \in \operatorname{Sp}(\mathcal{A})$, then

$$
\mathcal{A}_{l}(X, Y)=\mathcal{L}(X, Y) \text { and } \mathcal{A}_{r}(Y, X)=\mathcal{L}(Y, X) \text {, for every } Y .
$$

The following result can be derived easily from the definitions.

PROPOSITION 2.3. The classes $\mathcal{A}_{l}$ and $\mathcal{A}_{r}$ are semigroups in the sense of Definition 1.2. Moreover, $\mathcal{A}_{l} \subset \mathcal{A}_{+}$and $\mathcal{A}_{\tau} \subset \mathcal{A}_{-}$.

REMARK 2.4. The class $\mathcal{A}_{l} \cap \mathcal{A}_{r}$ is also a semigroup. We have $\mathcal{K}_{l} \cap \mathcal{K}_{\tau}=\mathcal{K}_{+} \cap \mathcal{K}_{-}$, the Fredholm operators, but an example in [9] shows that $\mathcal{W}_{l} \cap \mathcal{W}_{r} \neq \mathcal{W}_{+} \cap \mathcal{W}_{-}$.

Indeed, for every $T \in \mathcal{L}(X, Y)$ we consider the operator $T^{c o} \in \mathcal{L}\left(X^{* *} / X, Y^{* *} / Y\right)$, given by $T^{c o}(z+X)=T^{* *} z+Y$. Since $T \in \mathcal{W}_{+}$if and only if $T^{c o}$ is injective, and $T \in \mathcal{W}_{-}$if and only if $T^{c o}$ has dense range, $T \in \mathcal{W}_{+} \cap \mathcal{W}_{-}$whenever $T^{c o}$ is bijective.

On the other hand, for $X=\ell_{2}(J)$, where $J$ is the quasireflexive James' space, $X^{* *} / X$ is isomorphic to $\ell_{2}$ and $T \in \mathcal{L}(X)$ belongs to $\mathcal{W}_{l} \cap \mathcal{W}_{r}$ if and only if $T^{\text {co }}$ is regular with respect to the lattice structure in $\ell_{2}$. Since there are examples of operators $T \in \mathcal{L}(X)$ such that $T^{c o}$ is bijective but not regular, $\mathcal{W}_{l} \cap \mathcal{W}_{\tau} \neq \mathcal{W}_{+} \cap \mathcal{W}_{-}$.

We refer to [9] for further details.

The following result gives basic properties of $\mathcal{A}_{l}$ and $\mathcal{A}_{r}$.

Proposition 2.5. Let $S \in \mathcal{L}(Y, Z)$ and $T \in \mathcal{L}(X, Y)$.

(a) $S T \in \mathcal{A}_{l} \Rightarrow T \in \mathcal{A}_{l}$ and $S T \in \mathcal{A}_{r} \Rightarrow S \in \mathcal{A}_{r}$.

(b) For every $K \in \mathcal{A}(X, Y)$,

$$
T \in \mathcal{A}_{l} \Rightarrow T+K \in \mathcal{A}_{l} \quad \text { and } \quad T \in \mathcal{A}_{r} \Rightarrow T+K \in \mathcal{A}_{r}
$$


(c) $T \in \mathcal{A}^{d}{ }_{l} \Rightarrow T^{*} \in \mathcal{A}_{r}$ and $T \in \mathcal{A}^{d}{ }_{r} \Rightarrow T^{*} \in \mathcal{A}_{l}$. However, the converse implications are not true.

(d) $\mathcal{A}_{l}(X, Y) \neq \emptyset$ if and only if $\mathcal{A}_{r}(Y, X) \neq \emptyset$.

(e) $\mathcal{A}_{l}(X, Y)$ and $\mathcal{A}_{r}(X, Y)$ are open subsets of $\mathcal{L}(X, Y)$.

ProOF: We indicate the proof of some of the results. The remaining ones are direct consequences of the definitions.

(c) Let $J: c_{o} \rightarrow \ell_{\infty}$ be the natural inclusion. Then $J \notin \mathcal{K}_{l}$ because $c_{0}$ is not complemented in $\ell_{\infty}$. However, $N\left(J^{*}\right)$ is complemented by the lifting property of $\ell_{1}$; hence $J^{*} \in \Phi_{r}=\mathcal{K}_{r}$. Note that $\mathcal{K}=\mathcal{K}^{d}$ by Schauder's theorem.

(e) Let $T \in \mathcal{A}_{l}(X, Y)$ and $A \in \mathcal{L}(Y, X)$ such that $A T=I_{X}+K$ with $K \in \mathcal{A}(X)$. If $S \in \mathcal{L}(X, Y)$ and $\|A\| \cdot\|S\|<1$, then $I_{X}+A S$ is invertible, hence $A(T+S)=$ $I_{X}+A S+K \in \mathcal{A}_{l}$ by part (b); thus $T+S \in \mathcal{A}_{l}$ by part (a).

The concept of radical of an operator ideal is due to Pietsch [15, Section 4.3].

Definition 2.6: ([15]) The radical $\mathcal{A}^{\mathrm{rad}}$ of $\mathcal{A}$ is defined as follows:

$$
\mathcal{A}^{\mathrm{rad}}(X, Y):=\left\{K \in \mathcal{L}(X, Y): \begin{array}{l}
\text { for every } S \in \mathcal{L}(Y, X), \text { there exists } U \in \mathcal{L}(X) \\
\text { so that } I_{X}-U\left(I_{X}-S K\right) \in \mathcal{A}
\end{array}\right\}
$$

$\mathcal{A}^{\mathrm{rad}}$ is an operator ideal that contains $\mathcal{A}$. Moreover, $\operatorname{Sp}\left(\mathcal{A}^{\mathrm{rad}}\right)=\operatorname{Sp}(\mathcal{A})$ and $\mathcal{A}^{\mathrm{rad}}(X, Y)$ is always closed in $\mathcal{L}(X, Y)([15])$.

Lemma 2.7. In the definition of $\mathcal{A}^{\mathrm{rad}}$ we can write $I_{X}-\left(I_{X}-S K\right) U \in \mathcal{A}$ instead of $I_{X}-U\left(I_{X}-S K\right) \in \mathcal{A}$.

Proof: If $K \in \mathcal{A}^{\mathrm{rad}}$ and $L_{1}:=I_{X}-U\left(I_{X}-S K\right) \in \mathcal{A}$, then $I_{X}-U \in \mathcal{A}^{\mathrm{rad}}$. Thus there exists $W \in \mathcal{L}(X)$ so that $I_{X}-W U \in \mathcal{A}$, and

$$
\left(I_{X}-S K\right) U=W U\left(I_{X}-S K\right) U-L_{2}=W\left(I_{X}-L_{1}\right) U-L_{2}=I_{X}-L_{3}
$$

with $L_{2}, L_{3} \in \mathcal{A}$; hence $I_{X}-\left(I_{X}-S K\right) U \in \mathcal{A}$.

The converse implication is similar.

The best known example of radical is $\mathcal{I}:=\mathcal{F}^{\mathrm{rad}}$, the inessential operators. These operators were introduced by Yood [19] in the study of the perturbation of Fredholm operators. Kleinecke [11] introduced the name inessential operators and proved that $\mathcal{I}(X)$ is the biggest ideal of operators in $\mathcal{L}(X)$ with Riesz spectrum. 
REMARK 2.8. ([15, 4.3.8])

(a) $\mathcal{I}$ is contained in $\mathcal{A}^{\text {rad }}$ for every $\mathcal{A}$.

(b) $\mathcal{A} \subset \mathcal{B}$ implies $\mathcal{A}^{\mathrm{rad}} \subset \mathcal{B}^{\text {rad }}$ for every pair of operator ideals $\mathcal{A}$ and $\mathcal{B}$.

(c) $\left(\mathcal{A}^{\mathrm{rad}}\right)^{\mathrm{rad}}=\mathcal{A}^{\mathrm{rad}}$.

REMARK 2.9. In general, $\mathcal{A}^{\mathrm{rad}}$ is much bigger than $\mathcal{A}$. For example [7, Theorem 1], $\mathcal{L}(X, Y)=\mathcal{I}(X, Y)$ and $\mathcal{L}(Y, X)=\mathcal{I}(Y, X)$ in the following cases:

(i) $X$ contains no copies of $\ell_{\infty}$ and $Y=\ell_{\infty}$.

(ii) $X$ contains no complemented copies of $c_{0}$ and $Y=C[0,1]$;

(iii) $X$ contains no complemented copies of $\ell_{p}$ and $Y=L_{p}[0,1], 1 \leqslant p<\infty$.

Pietsch conjectured that the following question has a positive answer.

QUESTION 2.10. $([15,4.3 .7]) \quad$ Is $\mathcal{A}^{\mathrm{rad}}$ the biggest operator ideal $\mathcal{B}$ such that $\operatorname{Sp}(\mathcal{B})=\operatorname{Sp}(\mathcal{A}) ?$

As far as we know, the question is open even for $\mathcal{F}^{\mathrm{rad}}=\mathcal{I}$.

The semigroups associated to an operator ideal allow us to give a simpler description of the radical.

Proposition 2.11. For every $T \in \mathcal{L}(X, Y)$, the following assertions are equivalent:

(a) $T \in \mathcal{A}^{\mathrm{rad}}(X, Y)$.

(b) For every $S \in \mathcal{L}(Y, X), I_{X}-S T \in \mathcal{A}_{l}(X)$.

(b') For every $S \in \mathcal{L}(Y, X), I_{Y}-T S \in \mathcal{A}_{l}(Y)$.

(c) For every $S \in \mathcal{L}(Y, X), I_{X}-S T \in \mathcal{A}_{r}(X)$.

(c') For every $S \in \mathcal{L}(Y, X), I_{Y}-T S \in \mathcal{A}_{r}(Y)$.

ProOF: (a) $\Leftrightarrow$ (b). Assume $T \in \mathcal{A}^{\mathrm{rad}}(X, Y)$ and $S \in \mathcal{L}(Y, X)$. Then there exists $U \in \mathcal{L}(X)$ such that $K_{1}:=I_{X}-U\left(I_{X}-S T\right) \in \mathcal{A}$. Thus $U\left(I_{X}-S T\right)=I_{X}-K_{1} \in$ $\mathcal{A}_{l} \cap \mathcal{A}_{r}$, hence $I_{X}-S T \in \mathcal{A}_{l}$ (Proposition 2.5.a).

Conversely, if $I_{X}-S T \in \mathcal{A}_{l}$ for every $S \in \mathcal{L}(Y, X)$, then any left inverse modulo $\mathcal{A}$ of $I_{X}-S T$ can be taken as $U$ in Definition 2.6.

The proof of $(a) \Leftrightarrow(c)$ is similar to that of $(a) \Leftrightarrow(b)$, using Lemma 2.7.

(b) $\Leftrightarrow\left(b^{\prime}\right)$ and $(c) \Leftrightarrow\left(c^{\prime}\right)$. If $U \in \mathcal{L}(X)$ is a left (respectively, right) inverse of $I_{X}-S T$ modulo $\mathcal{A}$, then $I_{Y}+T U S$ is a left (respectively, right) inverse of $I_{Y}-T S$ modulo $\mathcal{A}$.

Corollary 2.12. $\quad \mathcal{L}(X, Y)=\mathcal{A}^{\mathrm{rad}}(X, Y) \Longleftrightarrow \mathcal{L}(Y, X)=\mathcal{A}^{\mathrm{rad}}(Y, X)$.

Let us see that the perturbation classes of the semigroups $\mathcal{A}_{l}, \mathcal{A}_{r}$ and $\mathcal{A}_{l} \cap \mathcal{A}_{r}$ coincide with the radical $\mathcal{A}^{\mathrm{rad}}$. 
THEOREM 2.13. Let $\mathcal{S}$ be one of the semigroups $\mathcal{A}_{l}, \mathcal{A}_{r}$ or $\mathcal{A}_{l} \cap \mathcal{A}_{s}$. If $\mathcal{S}(X, Y)$ is non-empty, then

$$
P \mathcal{S}(X, Y)=\mathcal{A}^{\mathrm{rad}}(X, Y) .
$$

Proof: We give the proof for $\mathcal{S}=\mathcal{A}_{l}$. The proof of the other cases is analogous.

Let $K \in \mathcal{A}^{\mathrm{rad}}(X, Y)$. For every $T \in \mathcal{A}_{l}(X, Y)$, we can select $A \in \mathcal{L}(Y, X)$ so that

$$
D_{1}:=I_{X}-A T \in \mathcal{A}(X) \text {. }
$$

For the operator $-A \in \mathcal{L}(Y, X)$, the definition of $\mathcal{A}^{\text {rad }}$ gives us another operator $U \in$ $\mathcal{L}(X)$ so that $D_{2}:=I_{X}-U\left(I_{X}+A K\right) \in \mathcal{A}$. Since $\mathcal{A} \subset P \mathcal{A}_{l}$ (Proposition 2.5.b),

$$
U A(T+K)=U\left(I_{X}-D_{1}+A K\right)=I_{X}-D_{2}-U D_{1} \in \mathcal{A}_{l} ;
$$

hence $T+K \in \mathcal{A}_{l}$. Thus $\mathcal{A}^{\mathrm{rad}}(X, Y) \subset P\left(\mathcal{A}_{l}\right)(X, Y)$.

For the converse inclusion, we show first that

$$
K \in P \mathcal{A}_{l}(X, Y), A \in \mathcal{L}(Y) \Rightarrow A K \in P \mathcal{A}_{l}(X, Y) .
$$

If $U \in \mathcal{A}_{l}(X, Y)$ and $A$ is invertible, then $U+A K=A\left(A^{-1} U+K\right) \in \mathcal{A}_{l}$; hence $A K \in$ $P \mathcal{A}_{l}$. For the general case it is enough to observe that every $A \in \mathcal{L}(Y)$ can be written as the sum of two invertible operators.

Now, let $K \in \mathcal{L}(X, Y), K \notin \mathcal{A}^{\mathrm{rad}}$. By Proposition 2.11, there exists $A \in \mathcal{L}(Y, X)$ such that $I_{X}-A K \notin \mathcal{A}_{l}(X)$.

Let $U \in \mathcal{A}_{l}(X, Y)$. Then $U\left(I_{X}-A K\right)=U-(U A) K \notin \mathcal{A}_{l}(X, Y)$. Therefore $(U A) K \notin P \mathcal{A}_{l}$, and (1) implies $K \notin P \mathcal{A}_{l}(X)$.

The semigroups $\mathcal{A}_{l}$ and $\mathcal{A}_{r}$ determine and are determined by $\mathcal{A}^{\mathrm{rad}}$.

Proposition 2.14. Let $\mathcal{A}$ and $\mathcal{B}$ be operator ideals. Then

$$
\mathcal{A}^{\mathrm{rad}}(X)=\mathcal{B}^{\mathrm{rad}}(X) \Longleftrightarrow \mathcal{A}_{l}(X)=\mathcal{B}_{l}(X) \Longleftrightarrow \mathcal{A}_{r}(X)=\mathcal{B}_{r}(X)
$$

Proof: Assume that $\mathcal{A}^{\mathrm{rad}}(X)=\mathcal{B}^{\mathrm{rad}}(X)$. Since $\mathcal{A} \subset \mathcal{A}^{\mathrm{rad}}=\left(\mathcal{A}^{\mathrm{rad}}\right)^{\mathrm{rad}}$ (Remark 2.8), $\mathcal{A}_{l} \subset \mathcal{A}^{\mathrm{rad}}$ l and $\mathcal{A}_{r} \subset \mathcal{A}^{\mathrm{rad}}{ }_{r}$. To prove the other two equalities it is enough to show that $\mathcal{A}^{\mathrm{rad}}{ }_{l}(X) \subset \mathcal{A}_{l}(X)$ and $\mathcal{A}^{\mathrm{rad}}{ }_{r}(X) \subset \mathcal{A}_{r}(X)$.

If $T \in \mathcal{A}^{\mathrm{rad}}{ }_{l}(X)$, then we can find $A \in \mathcal{L}(X)$ such that $I_{X}-A T \in \mathcal{A}^{\mathrm{rad}}$. By Theorem 2.13, $A T \in \mathcal{A}_{l}$; hence $T \in \mathcal{A}_{l}$.

The proof for $\mathcal{A}_{\boldsymbol{r}}$ is similar.

If $\mathcal{A}_{l}(X)=\mathcal{B}_{l}(X)$ or $\mathcal{A}_{r}(X)=\mathcal{B}_{r}(X)$, then it follows from Proposition 2.11 that $\mathcal{A}^{\mathrm{rad}}(X)=\mathcal{B}^{\mathrm{rad}}(X)$.

CoRollary 2.15. $\quad \mathcal{A}^{\mathrm{rad}}=\mathcal{B}^{\mathrm{rad}} \Longleftrightarrow \mathcal{A}_{l}=\mathcal{B}_{l} \Longleftrightarrow \mathcal{A}_{\tau}=\mathcal{B}_{r}$.

PROOF: It is enough to note that the proof of Proposition 2.14 shows that $\mathcal{A}(X)=$ $\mathcal{A}^{\text {rad }}(X)$ implies $\mathcal{A}_{l}(X, Y)=\mathcal{B}_{l}(X, Y)$ and $\mathcal{A}_{r}(Y, X)=\mathcal{B}_{r}(Y, X)$ for every $Y$; and $\mathcal{A}_{l}(X)=\mathcal{B}_{l}(X)$ or $\mathcal{A}_{r}(X)=\mathcal{B}_{r}(X)$ for every $X$ implies $\mathcal{A}^{\mathrm{rad}}(X, Y)=\mathcal{B}^{\mathrm{rad}}(X, Y)$ for every $X, Y$. 


\section{EXAMPLES}

Here we give information on the semigroups $\mathcal{A}_{l}$ and $\mathcal{A}_{r}$ in some cases. First we recall some useful characterisations of the inessential operators.

Theorem 3.1. $[14,1]$ For $K \in \mathcal{L}(X, Y)$ the following assertions are equivalent:

(a) $K$ is inessential.

(b) For every $S \in \mathcal{L}(Y, X)$ the kernel of $I_{X}-S K$ is finite dimensional.

$\left(\mathrm{b}^{\prime}\right)$ For every $S \in \mathcal{L}(Y, X)$ the kernel of $I_{Y}-K S$ is finite dimensional.

(c) For every $S \in \mathcal{L}(Y, X)$ the cokernel $X / \overline{R\left(I_{X}-S K\right)}$ is finite dimensional.

(c') For every $S \in \mathcal{L}(Y, X)$ the cokernel $Y / \overline{R\left(I_{Y}-K S\right)}$ is finite dimensional.

QUESTION 3.2. Is it possible to find characterisations of $\mathcal{A}^{\mathrm{rad}}$ similar to Theorem 3.1 for other semigroups $\mathcal{A}$ ?

Recall that a Banach space $X$ is said to have the Dunford-Pettis property if $\mathcal{W}(X, Y) \subset \mathcal{C C}(X, Y)$ for every Banach space $Y$. The spaces $C(K)$ and $L_{1}(\mu)$ have the Dunford-Pettis property. We refer to [6] for additional information.

Proposition 3.3. Assume that $X$ or $Y$ has the Dunford-Pettis property. Then

$$
\mathcal{W}_{l}(X, Y)=\Phi_{l}(X, Y) \quad \text { and } \quad \mathcal{W}_{r}(X, Y)=\Phi_{r}(X, Y)
$$

Proof: Recall that $\mathcal{K}_{l}=\Phi_{l}$ and $\mathcal{K}_{r}=\Phi_{r}$. If $X$ has the Dunford-Pettis property and $T \in \mathcal{W}(X, Y)$, then $S T \in(\mathcal{W} \cap \mathcal{C C})(X)$ for every $S \in \mathcal{L}(Y, X)$. Thus $(S T)^{2} \in \mathcal{K}$. In particular, $\operatorname{dim} N(I-S T)<\infty$. By Theorem 3.1, $T$ is inessential. Hence,

$$
\mathcal{K}(X, Y) \subset \mathcal{W}(X, Y) \subset \mathcal{I}(X, Y)
$$

Since $\mathcal{K}_{l}=\mathcal{I}_{l}$, it follows from Corollary 2.15 that $\mathcal{W}_{l}(X, Y)=\mathcal{K}_{l}(X, Y)$.

The proof of the other cases is analogous.

Corollary 3.4. If $X$ has the Dunford-Pettis property and $Y$ is a noncomplemented closed subspace of $X$, then the inclusion $J_{Y}: Y \rightarrow X$ does not belong to $\mathcal{W}_{l}$

Proof: Clearly, $J_{Y} \notin \Phi_{l}(Y, X)=\mathcal{W}_{l}(Y, X)$.

REMARK 3.5. The Hardy space $H^{1}$ is a closed non-complemented subspace of $L^{1}$. Since $L^{1}$ has the Dunford-Pettis property, it follows from the previous corollary that the inclusion of $H^{1}$ into $L^{1}$ does not belong to $\mathcal{W}_{l}$. This result was previously established in [4; Lemma 8] using techniques of harmonic analysis.

Apart from the inessential operators, we can show other examples of operator ideals which coincide with its radical. 
Definition 3.6: We say that an operator ideal $\mathcal{A}$ is radical if $\mathcal{A}=\mathcal{A}^{\mathrm{rad}}$.

DEFINITION 3.7: Let $Z_{0}$ be a fixed infinite dimensional Banach space. An operator $T \in \mathcal{L}(X, Y)$ belongs to the class $Z_{0}-\mathcal{N C}$ if there is no closed subspace $M$ of $X$ isomorphic to $Z_{0}$ so that the restriction $\left.T\right|_{M}$ is an isomorphism, and $T(M)$ is complemented in $Y$.

For some spaces, like $Z_{0}=\ell_{1} \oplus \ell_{2}$, the identity $I_{Z_{0}}$ is not in $Z_{0}-\mathcal{N C}$, but we can write it as the sum of two projections that are both in $Z_{0}-\mathcal{N C}$. Thus $Z_{0}-\mathcal{N C}\left(Z_{0}\right)$ is not a subspace of $\mathcal{L}\left(Z_{0}\right)$. However, we shall give examples of spaces so that $Z_{0}-\mathcal{N C}$ is an operator ideal.

PRoposit ION 3.8. Let $Z_{0}$ be a fixed infinite dimensional Banach space. Then

(a) $Z_{0}-\mathcal{N C}$ satisfies properties $\left(\alpha_{1}\right)$ and $\left(\alpha_{3}\right)$ in Definition 1.1. Moreover, $I_{X} \in Z_{0}-\mathcal{N C} \Longleftrightarrow X$ contains no complemented copy of $Z_{0}$.

(b) Suppose that $Z_{0}-\mathcal{N C}$ is an operator ideal. Then every operator ideal $\mathcal{A}$ that satisfies $\operatorname{Sp}(\mathcal{A})=\operatorname{Sp}\left(Z_{0}-\mathcal{N C}\right)$ is contained in $Z_{0}-\mathcal{N C}$. In particular, $Z_{0}-\mathcal{N C}$ is a radical operator ideal.

Proof: (a) Since $Z_{0}$ is infinite dimensional, the finite rank operators are contained in $Z_{0}-\mathcal{N C}$. So it satisfies property $\left(\alpha_{1}\right)$.

To prove property $\left(\alpha_{3}\right)$, observe that for every operator $T \in \mathcal{L}(X, Y)$, if the restriction $\left.T\right|_{M}$ on a closed subspace $M$ of $X$ is an isomorphism and $T(M)$ is complemented in $Y$, then $M$ is complemented in $X$. Indeed, if $Y=N \oplus T(M)$, then $X=T^{-1}(N) \oplus M$.

We take $B \in \mathcal{L}(W, X), T \in \mathcal{L}(X, Y)$ and $A \in \mathcal{L}(Y, Z)$, and we suppose that $A T B \notin$ $Z_{0}-\mathcal{N C}(W, Z)$. Then there exists a closed subspace $M$ of $W$ isomorphic to $Z_{0}$ so that $\left.A T B\right|_{M}$ is an isomorphism and $(A T B)(M)$ is complemented in $Z$. Let $N$ be a closed subspace of $Z$ such that $Z=N \oplus(A T B)(M)$. Then $Y=A^{-1}(N) \oplus(T B)(M)$. Moreover; $B(M)$ is isomorphic to $Z_{0}$ and $\left.T\right|_{B(M)}$ is an isomorphism; hence $T \notin Z_{0}-\mathcal{N C}$. Thus $Z_{0}-\mathcal{N C}$ satisfies property $\left(\alpha_{3}\right)$.

The second assertion of part (a) is immediate.

(b) Note that $Z_{0} \notin \operatorname{Sp}(\mathcal{A})=\operatorname{Sp}\left(Z_{0^{-}} \mathcal{N C}\right)$, by part (a). Suppose that $T \in \mathcal{L}(X, Y)$, but $T \notin Z_{0}-\mathcal{N C}$. Then there exists a subspace $M$ of $X$ isomorphic to $Z_{0}$ such that $\left.T\right|_{M}$ is an isomorphism and both $M$ and $T(M)$ are complemented. Thus we can find $A \in \mathcal{L}\left(Y, Z_{0}\right)$ and $B \in \mathcal{L}\left(Z_{0}, X\right)$ so that $A T B=I_{Z_{0}}$; hence $T \notin \mathcal{A}$.

Let us see that the class of all operators preserving no complemented copies of one of the spaces $\ell_{p}, 1 \leqslant p \leqslant \infty$ or $c_{0}$ is a radical operator ideal.

THEOREM 3.9. Suppose that $Z_{0}$ is one of the spaces $\ell_{p}, 1 \leqslant p \leqslant \infty$, or $c_{0}$. Then $Z_{0}-\mathcal{N C}$ is a radical operator ideal.

ProOF: By Proposition 3.8, we only have to show that $Z_{0}-\mathcal{N C}(X, Y)$ is a subspace of $\mathcal{L}(X, Y)$. In order to do that, let $S \in Z_{0}-\mathcal{N C}(X, Y)$ and $T \in \mathcal{L}(X, Y)$, and assume 
that $M$ is a subspace of $X$ isomorphic to $Z_{0}$ so that $\left.(S+T)\right|_{M}$ is an isomorphism and $(S+T)(M)$ is complemented in $Y$. We have to show that $T \notin Z_{0}-\mathcal{N C}(X, Y)$.

First we assume that $Z_{0}$ is $\ell_{p}$ with $1 \leqslant p<\infty$, or $c_{0}$. Let $P$ be a projection on $Y$ such that $R(P)=(S+T)(M)$. Then $\left.P S\right|_{M}+\left.P T\right|_{M}=\left.(S+T)\right|_{M}$, and by Proposition $3.8(\mathrm{a}),\left.P S\right|_{M} \in Z_{0}-\mathcal{N C}$.

We claim that $\left.P S\right|_{M} \in \mathcal{S S}$; that is, it is strictly singular. Indeed, otherwise we could find a closed, infinite dimensional subspace $M_{1}$ of $M$ such that $\left.P S\right|_{M_{1}}$ is an isomorphism. By [13, Proposition 2.a.2], $P S\left(M_{1}\right)$ contains a subspace $N_{2}$ which is isomorphic to $Z_{0}$ and complemented in $R(P)$. Since we can write $N_{2}=P S\left(M_{2}\right)$, where $M_{2}$ is a closed subspace of $M_{1},\left.P S\right|_{M_{2}} \notin Z_{0}-\mathcal{N C}(X, Y)$, a contradiction.

Note that $\left.(S+T)\right|_{M} \in \mathcal{K}_{l}(M, Y)$, because it is an isomorphism with complemented range (see Example 2.2). Moreover, $\mathcal{S S} \subset \mathcal{I}$ and $\mathcal{I}$ is the perturbation class of $\mathcal{K}_{l}$ [15]. Thus $\left.P T\right|_{M} \in \mathcal{K}_{l}$.

So, there exists a finite codimensional closed subspace $N$ of $M$ (which is isomorphic to $M$ ) such that $\left.P T\right|_{N}$ is an isomorphism and $P T(M)=P T(N)$. Thus $T \notin Z_{0}-\mathcal{N C}$.

In the case $Z_{0}=\ell_{\infty}$, we can repeat a similar argument, using three facts. First, that every subspace of a Banach space isomorphic to $\ell_{\infty}$ is complemented; second, that for every non-weakly compact operator $R: \ell_{\infty} \rightarrow Z$, there exists a subspace $M$ of $\ell_{\infty}$ isomorphic to $\ell_{\infty}$ so that $R \mid M$ is an isomorphism [13, Proposition 2.f.4]; and third, that every weakly compact $R: \ell_{\infty} \rightarrow Z$ is inessential [7, Theorem 1 ].

REMARK 3.10. (a) Theorem 3.9 gives a positive answer to Question 2.10 for $Z_{0}-\mathcal{N C}$, when $Z_{0}$ is one of the spaces $\ell_{p}, 1 \leqslant p \leqslant \infty$ or $c_{0}$.

(b) It follows from the results of Bessaga and Pelczynski in [5] (see [16, Lemma 9.2 in Chapter C.II.]) that the conjugate $T^{*}$ of $T \in \mathcal{L}(X, Y)$ is unconditionally converging, that is, $T \in \mathcal{U C}^{d}$, if and only if $X$ has no closed subspace $N$ isomorphic to $\ell_{1}$ such that $T J_{N}$ is an isomorphism and $T(N)$ is complemented in $Y$.

Thus, it follows from Theorem 3.9 that $\mathcal{U} \mathcal{C}^{d}$ is the radical operator ideal $\ell_{1}-\mathcal{N C}$.

\section{REFERENCES}

[1] P. Aiena and M. González, 'Essentially incomparable Banach spaces and Fredholm theory', Proc. Roy. Irish Acad. Sect. A 93 (1993), 49-59.

[2] P. Aiena, M. González, and A. Martínez-Abejón, 'Operator semigroups in Banach space theory', Boll. Un. Mat. Ital. Sez. B Artic. Ric. Mat. (8) 4 (2001), 157-205.

[3] F. Atkinson, 'Relatively regular operators', Acta Sci. Math. (Szeged) 15 (1953), 38-56.

[4] K. Astala and H.-O. Tylli, 'Seminorms related to weak compactness and to tauberian operators', Math. Proc. Cambridge Philos. Soc. 107 (1990), 365-375.

[5] C. Bessaga and A. Pelczyński, 'On basis and unconditional convergence of series in Banach spaces', Studia Math. 17 (1958), 151-164.

[6] J. Diestel, 'A survey of results related to the Dunford-Pettis property', Contemp. Math. 2 (1980), 15-60. 
[7] M. González, 'On essentially incomparable Banach spaces', Math. Z. 215 (1994), 621-629.

[8] M. González and A. Martínez-Abejón, 'Lifting unconditionally converging series and semigroups of operators', Bull. Austral. Math. Soc. 57 (1998), 135-146.

[9] M. González, E. Saksman and H.-O. Tylli, 'Representing non-weakly compact operators', Studia Math. 113 (1995), 265-282.

[10] N. Kalton and A. Wilansky, 'Tauberian operators in Banach spaces', Proc. Amer. Math. Soc. 57 (1976), 251-255.

[11] D. Kleinecke, 'Almost-finite, compact, and inessential operators', Proc. Amer. Math. Soc. 14 (1963), 863-868.

[12] A. Lebow and M. Schechter, 'Semigroups of operators and measures of noncompactness', J. Funct. Anal. 7 (1971), 1-26.

[13] J. Lindenstrauss and L. Tzafriri, Classical Banach spaces I. Sequence spaces (Springer-Verlag, Berlin, Heidelberg, New York, 1977).

[14] A. Pietsch, 'Inessential operators in Banach spaces', Integral Equations Operator Theory 1 (1978), 589-591.

[15] A. Pietsch, Operator ideals (North-Holland, Amsterdam, New York, Oxford, 1980).

[16] D. Przeworska-Rolewicz and S. Rolewicz, Equations in linear spaces (P.W.N., Warszawa, 1968).

[17] D.G. Tacon, 'Generalized semi-Fredholm transformations', J. Austral. Math. Soc. Ser. A 34. (1983), 60-70.

[18] K.W. Yang, 'Operators invertible modulo the weakly compact operators', Pacific J. Math. 71 (1977), 559-564.

[19] B. Yood, 'Difference algebras of linear transformations on a Banach space', Pacific J. Math. 4 (1954), 615-636.

Dipartimento di Matematica ed Applicazioni

Università di Palermo

Viale delle Scienze

I-90128 Palermo

Italy

Departamento de Matemáticas

Universidad de Oviedo

E-33007 Oviedo

Spain

\author{
Departamento de Matemáticas \\ Universidad de Cantabria \\ E-39071 Santander \\ Spain
}

